\title{
Improved Early Recognition of Coronavirus Disease-2019 (COVID-19): Single-Center Data from a Shanghai Screening Hospital
}

\author{
Ling Peng, $\mathrm{MM}^{1,2 *}$; Kang-Yong Liu, $\mathrm{MD}^{3 *}$; Fei Xue, $\mathrm{MB}^{2}$; Ya-Fang Miao, $\mathrm{MM}^{2}$; Ping-An Tu, $\mathrm{MM}^{4}$; Chao Zhou, $\mathrm{MM}^{2 *}$ \\ 'Guizhou medical university, Guiyang, China. No. 9 Beijing Road, Yunyan District, Guiyang 550025, China \\ ${ }^{2}$ Department of Respiratory Medicine, Zhoupu Hospital affiliated to Shanghai University of Medicine and Health Sciences, Shanghai, China. \\ No. 1500 Zhouyuan Road, Pudong new District, Shanghai 201318, China \\ 'Department of Neurology, Zhoupu Hospital affiliated to Shanghai University of Medicine and Health Sciences, Shanghai, China. No. 1500 \\ Zhouyuan Road, Pudong new District, Shanghai 201318, China \\ ${ }^{4}$ Faculty office, Zhoupu Hospital affiliated to Shanghai University of Medicine and Health Sciences. No. 1500, Zhouyuan Road, Pudong New \\ District, Shanghai 201318, China
}

\begin{abstract}
Background: In December 2019, an outbreak of a novel coronavirus disease (COVID-19; previously known as 2019-nCoV) was reported in Wuhan, Hubei province, China, which has subsequently affected more than 200 countries worldwide including Europe, North America, Oceania, Africa and other places. The number of infected people is rapidly increasing, while the diagnostic method of COVID-19 is only by nucleic acid testing.

Objective: To explain the epidemiological characteristics, clinical features, imaging manifestations and to judge diagnostic value of COVID-19 by analyzing the clinical data of COVID-19 suspected and confirmed patients in a non-outbreak, Shanghai, China. To clarify the early epidemiology and clinical characteristics about COVID-19.

Methods: Cross-sectional, single-center case reports of the 86 patients screened at Zhoupu Hospital in Pudong New District, Shanghai, China, from January 23 to February 16, 2020. Epidemiology, demography, clinical, laboratory and chest CTs were collected and analyzed. The screened patients were divided into COVID-19 and non-COVID-19 based on nucleic acid test results.

Results: Of the 86 screened patients, 11 were confirmed (12.8\%) by nucleic acid testing (mean age $40.73 \pm 11.32,5$ males). No significant differences were found in clinical symptoms including fever, cough, dyspnea, sore throat, and fatigue $(P>0.05)$. No statistical difference was observed in plasma C-reactive protein (CRP) between the two groups (COVID-19 and non-COVID-19) of patients $(P=0.402)$, while the white blood cell count and lymphocyte count of the confirmed patients were slightly lower than those of the suspected patients $(P<0.05)$. Some non-COVID-19 chest CTs also showed subpleural lesions, such as ground-glass opacities (GGO) combined with bronchiectasis; or halo nodules distributed under the pleura with focal GGO; consolidation of subpleural distribution or combined with air bronchi sign and vascular bundle sign, etc.

Conclusion: The early clinical manifestations and imaging findings of COVID-19 are not characteristic in non-outbreak areas. Etiological testing should be performed as early as possible for clinically suspected patients.

Keywords: Clinical characteristics, Computed tomographic, Coronavirus, COVID-19, Epidemic

Cite this article as: Peng L, Liu KY, Xue F, Miao YF, Zhou C. Improved early recognition of coronavirus disease-2019 (COVID-19): single-center data from a shanghai screening hospital. Arch Iran Med. 2020;23(4):272-276. doi: 10.34172/aim.2020.10.
\end{abstract}

Received: March 16, 2020, Accepted: March 21, 2020, ePublished: April 1, 2020

\section{Introduction}

In December 2019, an outbreak of severe acute respiratory syndrome coronavirus 2 (SARS-CoV-2) infection appeared in Wuhan, Hubei province, China. On February 12,2020 , the WHO officially named the disease caused by this new type of coronavirus as "Coronavirus disease 2019" (COVID-19). ${ }^{1}$ SARS-CoV-2 is a new coronavirus that has not been found in humans before. The highly infectious virus has spread rapidly, and has no specific treatment, posing a huge threat to global public health. ${ }^{2}$ SARS-CoV-2 is a type of single-stranded positive-stranded RNA virus; its outer membrane has prominent stickshaped protruding particles. The particles are round or oval, always polymorphic with a diameter of $60-140$ nm particles, 9-12 nm long surface rod-shaped particles under the electron microscope. ${ }^{3}$ Its genetic characteristics are significantly different from those of SARSr-CoV and MERSr-CoV. ${ }^{4}$ The viral genome analysis reveals that the virus shares $88 \%$ sequence identity with two batderived severe acute respiratory syndrome (SARS)-related coronaviruses. ${ }^{5}$ Using in vitro isolation and culture, 2019$\mathrm{nCoV}$ can be found in human respiratory epithelial cells in about 96 hours, while it takes about 6 days to isolate and culture in Vero E6 and Huh-7 cell lines.

Based on current epidemiological investigations, the incubation period of neo-coronary pneumonia is 1-14 days, mostly 3-7 days. Fever, dry cough, and fatigue are the main manifestations. A few patients also have

\footnotetext{
\# These authors contributed to the work equally and be regarded as co-first anthers.

*Corresponding Author: Chao Zhou, MM; 1500 Zhouyuan Road, Pudong new District, Shanghai, 201318, China. Tel: +86-21-68135590,

Email: zhou2000sh@163.com
} 
nasal congestion, runny nose, sore throat, myalgia, and diarrhea. Most of the infected people are mild patients; however, some severe patients usually have dyspnea and/ or hypoxemia one week after the onset of illness. In severe cases, they could quickly progress to acute respiratory distress syndrome, septic shock, severe metabolic acidosis, coagulopathy and multi-organ failure. ${ }^{6}$

At present, real-time reverse-transcription-polymerasechain-reaction (RT-PCR) is still the only way to clearly diagnose COVID-19.7 The high false negative rate of nucleic acid tests and the shortage of kits limit timely diagnosis of COVID-19 in the early stages of the outbreak. ${ }^{8}$ Chest CT can identify the early phase lung infection. It is the thin slice chest CT that plays an important role in combating infectious disease. ${ }^{9}$ We mainly analyze the clinical features and imaging manifestations of 86 patients including 11 COVID-19 and 74 non-COVID-19 cases diagnosed in a fever clinic in Shanghai, China.

\section{Materials and Methods}

Study Design and Inclusion Population

Through cross-sectional studies, clinically suspected patients who were admitted to the Zhoupu Hospital, Pudong New District, Shanghai from January 23, 2020 to February 16, 2020 were included. The patients were divided into COVID-19 and non-COVID-19 in accordance with "China National Health Commission, Diagnosis and treatment of pneumonia caused by new coronavirus infection (version 5) (February 8, 2020)" (Table 1).

Nasopharyngeal swab samples were collected for realtime PCR test, while basic information was obtained from the electronic medical record system. We recorded the patients' symptoms, signs, contact history, potential comorbidities, laboratory test results on the first day, imaging (chest CT), the time for came to Shanghai, selfisolation time, etc. All enrolled patients provided their informed consent.
Statistical Analysis

The statistical software SPSS 25.0 was used for summary analysis. The data that met the normal distribution after normality test were expressed in means \pm standard deviations, and $t$ test was used to compare differences between groups; the data with non-normal distribution were expressed in medians and interquartile range, and non-parametric tests were used for comparison; $\chi^{2}$ test was used for paired count data. A two-tailed $P<0.05$ was considered statistically significant.

\section{Results}

\section{Epidemiology}

Until February 16, 2020, 331 cases of COVID-19 were confirmed in Shanghai, including 172 males and 159 females; the oldest were 88 years old and the youngest was 7 months old; 144 had a history of living in or traveling to Hubei, China; 154 had a history of contact with COVID-19 patients.

We collected a total of 86 patients who were sent to our hospital for screening during the three weeks from January 23, 2020 to February 16, 2020, of whom 48 (55.8\%) came from other provinces (including 37 Hubei, 2 Chongqing, 3 Henan, 3 Jiangsu, 2 Jiangxi, and 1 Anhui).

Eleven patients (12.8\%) were diagnosed by nucleic acid testing (mean age $40.73 \pm 11.32$ years, 5 males), of whom $7(63.6 \%)$ had a history of travel to Hubei (5 Wuhan, 1 Huanggang, 1 Xiaogan), 2 patients had close contact with the COVID-19 patients, and 2 taxi drivers. A total of 74 patients were non-COVID-19 (mean age $39.67 \pm 13.90$ years, 34 men).

\section{Clinical Symptom Spectrum}

The two groups of patients were analyzed statistically for fever $(P=0.184 ; 95 \% \mathrm{CI}-0.47,0.09)$, cough $(P=0.69$; $95 \%$ CI $-0.25,0.39)$, and dyspnea $(P=0.199 ; 95 \%$ CI $-0.07,0.34)$, sore throat $(P=0.121 ; 95 \% \mathrm{CI}-0.06,0.52)$, fatigue $(P=0.591 ; 95 \% \mathrm{CI}-0.44,0.26)$ and there were no

Table 1. Suspected cases: Any one (or none) of the items in the epidemiological history and two of the clinical manifestations; Confirmed cases: Etiological in the suspected cases with one of the etiologies

(1) Travel history or residence history in Wuhan province and surrounding areas, or other communities with
reported cases within 14 days before the onset of illness;
(2) History of contact with novel coronavirus infected person (nucleic acid test positive) within 14 days before
the onset of illness;
(3) Contact with people who come from Wuhan province and surrounding areas or communities with
reported cases within 14 days before the onset of illness;
(4) Cluster onset.
(1) Fever and/or respiratory symptoms;
Clinical manifestation
(2) The total number of white blood cells is normal or decreased in the early stage of onset, or the lymphocyte
Count is reduced.
(1) Real-time RT-PCR to detect novel coronavirus nucleic acid positive;
(2) Viral gene sequencing, highly homologous to known novel coronaviruses

RT-PCR, reverse-transcription-polymerase-chain-reaction. 
significant differences in these clinical manifestations $(P>$ 0.05). Comparing the laboratory testing indicators on the day of the patient's consultation, there was no statistical difference in the plasma C-reactive protein (CRP) between the two groups of patients $(P=0.402)$, while the white blood cell count and lymphocyte count of the confirmed patients were slightly lower than those of the suspected patients $(P<0.05)$ (Table 2$)$.

\section{CT Imaging Findings}

We compared and analyzed the imaging features of COVID-19 and non-COVID-19. The early CT imaging features of 11 confirmed COVID-19 patients were mainly patchy ground-glass opacities (GGO) and consolidation shadows, and they were mostly distributed along the bronchovascular bundles and subpleural space, with a thickened leaflet interval and thickened lobular septum, showing "crazy-paving pattern" and vascular bundle sign (Figure 1); some patients showed isolated nodular lesions with halo signs in subpleural space (Figure 2).

In non-COVID-19 patients, CT imaging of the lungs showed bronchiolar wall thickening, local bronchiectasis, peripheral exudative inflammation, and tree-in-bud pattern (Figure 3); consolidation with bronchial dilatation distributed along the lung segment or lung lobe, and antihalo sign could be seen in some cases; or characterized by a mixture of GGO combined with bronchiectasis, which are not in subpleural space and distributed along the bronchial vascular bundle (Figure 4).

However, it is difficult to distinguish between nonCOVID-19 and COVID-19 in some imaging findings, such as combination of GGO distributed under the pleura with bronchiectasis; subpleural halo nodules with focal GGO; consolidation with air bronchioles or subpleural vascular bundle sign (Figure 5).

\section{Discussion}

COVID-19 is a self-limiting disease, but it has also developed into a fatal disease. Respiratory droplets and close contact transmission are currently considered to be its main route of transmission. In addition, there is a possibility of aerosol transmission in a relatively closed environment when exposed to high concentrations of aerosol for a long time. ${ }^{6}$

Table 2. Demographic and Baseline Characteristics of COVID-19 Confirmed and Suspected Patients

\begin{tabular}{|c|c|c|c|c|c|}
\hline & Number of Cases (n) & Suspected Diagnosis $(n=75)$ & Confirmed Diagnosis $(n=11)$ & $\chi^{2} / \mathrm{t}$ & $P$ Value \\
\hline Gender: Male/Female & $39 / 47$ & $34 / 40$ & $5 / 6$ & 0.029 & 0.977 \\
\hline Age & & & $40.73 \pm 11.32$ & -0.281 & 0.782 \\
\hline Onset to treatment (days) & & $3.57 \pm 3.91$ & $3.18 \pm 4.00$ & 0.3 & 0.769 \\
\hline \multicolumn{6}{|l|}{ Symptom } \\
\hline Fever, No. (\%) & & $54(72 \%)$ & $10(91 \%)$ & -1.341 & 0.184 \\
\hline Systemic soreness, No. (\%) & & $41(55 \%)$ & $7(64 \%)$ & -0.551 & 0.591 \\
\hline Cough, No. (\%) & & $46(61 \%)$ & $6(55 \%)$ & 0.406 & 0.692 \\
\hline Dyspnea, No. (\%) & & $10(13 \%)$ & $0(0 \%)$ & 1.295 & 0.199 \\
\hline Sore throat, No. (\%) & & $24(32 \%)$ & $1(9 \%)$ & 1.567 & 0.121 \\
\hline Runny nose, No. (\%) & & $6(8 \%)$ & $0(0 \%)$ & 0.967 & 0.337 \\
\hline \multicolumn{6}{|l|}{ Laboratory inspection } \\
\hline White blood cell count $\left(\times 10^{9} / \mathrm{L}\right)$ & & $7.61 \pm 2.43$ & $6.41 \pm 1.57$ & 2.175 & 0.043 \\
\hline Lymphocyte count (× 10/L) & & $2.32 \pm 3.16$ & $1.43 \pm 0.60$ & 2.189 & 0.032 \\
\hline $\mathrm{CRP}(\mathrm{mg} / \mathrm{L})$ & & $10.00 \pm 13.75$ & $7.66 \pm 7.33$ & 0.854 & 0.402 \\
\hline
\end{tabular}

No. (\%), where $\mathrm{N}$ is the total number of patients with available data.

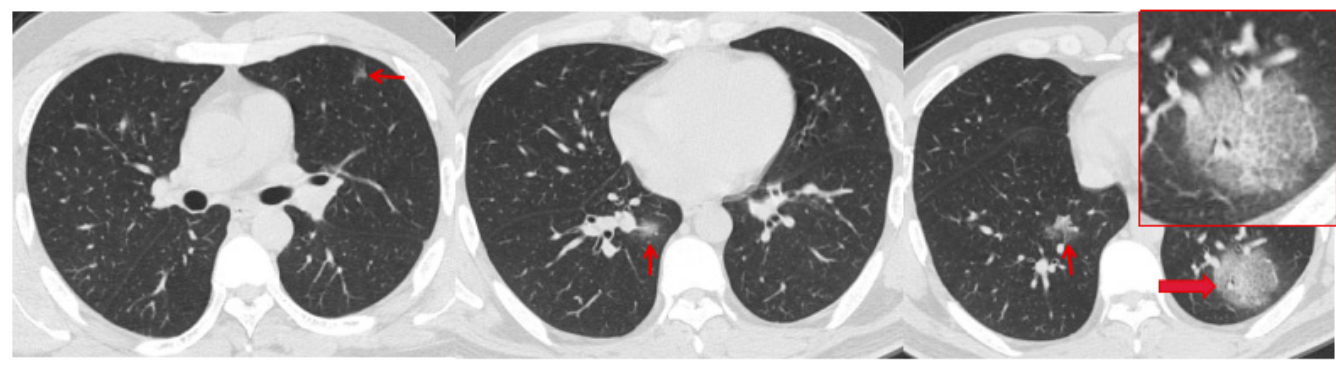

Figure 1. Transverse Thin-Section CT Scans in Patients with COVID-19 Pneumonia. 32-year-old woman, from Xiaogan of Hubei, China, came to Shanghai on January 23, 2020, on February 2, 2020, she was evaluated for fever, cough, and fatigue for 1 day. The nasopharyngeal test strip was positive for nucleic acid test. CT shows multiple ground-glass opacity (GGO) (red arrow) under the pleura, ground-glass opacity nodule shadow in the left lower lobe, in which "crazy-paving pattern" and vascular bundles sign can be seen (red thick arrow). 


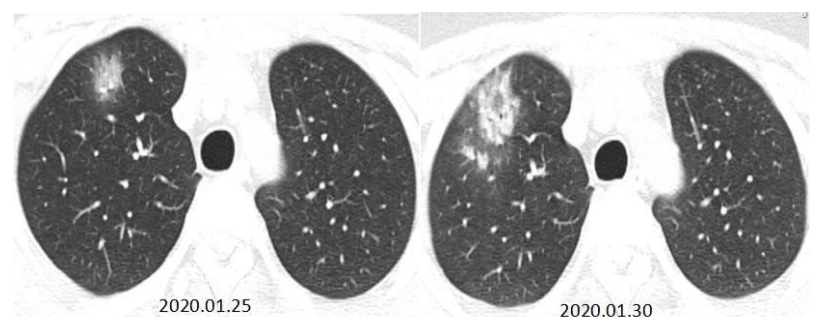

Figure 2. Transverse Thin-Section CT Scans in Patients With COVID-19 Pneumonia. 22-year-old man, from Huanggang of Hubei, came to Shanghai on January 25, 2020. He was evaluated on January 25, 2020 for fever, cough, and fatigue for 2 days, and the third nucleic acid test was positive, CT shows isolated patchy lesions with halo nodule in subpleural space of the right upper lung (January 25, 2020). Review of CT after 5 days revealed that the patchy lesions were enlarged associated with air bronchograms and anti-halo sign (2020.01.30).

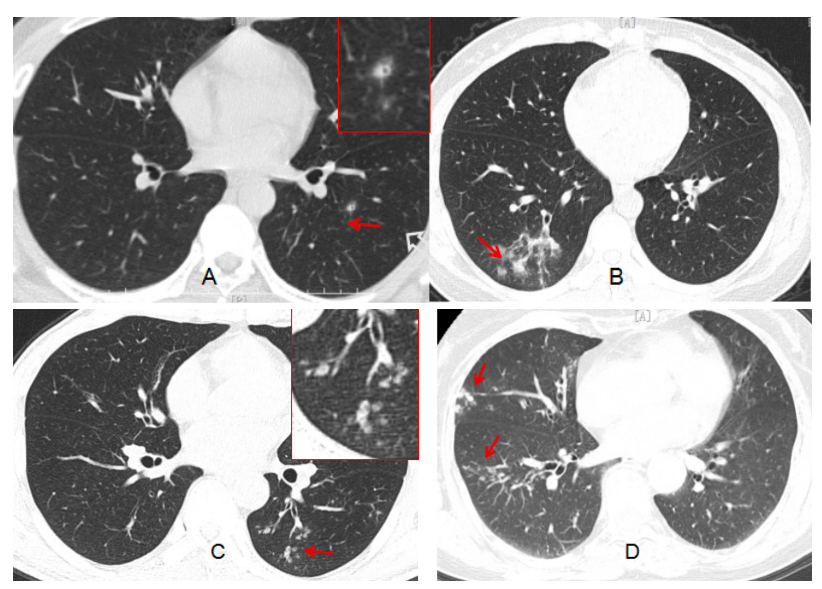

Figure 3. Non-COVID-19 patients, Characterized by Internal Bronchovascular Bundle Thickening, Local Bronchiectasis, with Surrounding Exudative Inflammation and Tree-in-Bud Pattern. (A) 31-yearold man with fever for 2 days; CT shows bronchial wall thickening of the left lower lobe and surrounding ground-glass nodule (red arrow), partial enlarged image (red box); (B) 34-year-old woman, with fever, cough for 6 days; CT shows bronchiectasis of the right lower lobe and its expansion peripheral ground-glass opacities (red arrow); (C) 40-year-old man, coughing for 1 week; CT shows multiple central leaflet nodules in the left lower lobe, with tree-in-bud pattern (red arrow); (D) 68-year-old woman with fever for 1 day; CT shows bronchiectasis of the right middle lobe, multiple central nodules under the pleura and lower lobe in the lateral middle lobe indicated as tree-in-bud pattern (red arrows).

This study first analyzed the clinical features of COVID-19 and Non-COVID-19 patients. From January 23, 2019 to February 16, 2020, a total of 86 people, which are divided into COVID-19 and non-COVID-19 based on nucleic acid test results were screened for outpatient visits. A total of $11(12.8 \%)$ patients had COVID-19 pneumonia, of whom 7 (63.6\%) had a history of travel to Hubei, 10 (90.9\%) had fever, 7 (63.6\%) had fatigue, and $6(54.5 \%)$ had cough. No dyspnea or severe hypoxemia was found in 11 patients, which may be related to the patients who are in the early stage of infection or belong to the common type. ${ }^{6}$

After a comparative analysis of the clinical characteristics of the two groups of patients, we found that the general

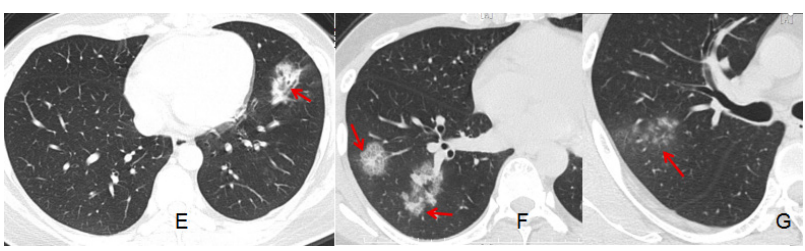

Figure 4. Non-COVID-19 Patients, Day 2 after Symptom Onset. CT shows consolidation with air bronchioles sign along the lung segment or lobe (E). A 28-year-old man with fever, fatigue for 2 days; CT shows multiple pulmonary segmental consolidation with anti-halo sign in the right lower lobe (F). A 37-year-old male patient with fever, fatigue, and cough for 1 day; CT shows ground-glass opacities (GGO) along with bronchovascular bundles $(\mathbf{G})$

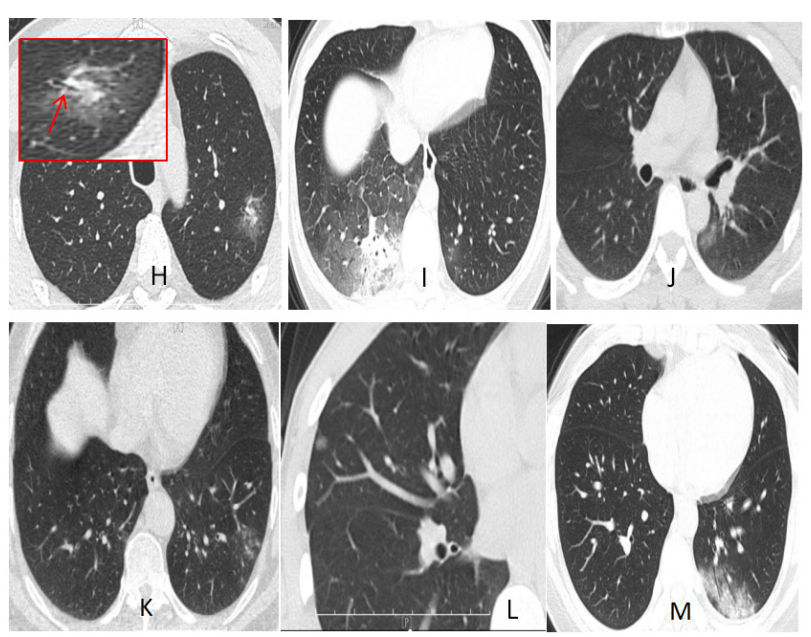

Figure 5. Non-COVID-19 Patients. CT shows mixed GGO with bronchiectasis of the left upper lobe in subpleura, partial enlarged picture $(\mathbf{H}$ red box); mixed GGO with bronchiectasis and a thickened leaflet interval of the right lower lobe subpleurally (I); lung window showing GGO in the dorsal segment of the right lower lobe (J); Multiple patchy lesions under the right lower lobe with halo sign (K); small nodular lesions can be seen outside right middle lobe segmental subpleurally (L); consolidation with air bronchioles and vascular bundle sign in the left lower lobe subpleurally (M).

clinical manifestations are not specific. Hematological examination shows that the white blood cell count and lymphocyte count of confirmed patients are slightly lower than those of suspected patients, which is consistent with the National Health Commission of the People's Republic of China "Diagnosis and Treatment of New Coronavirus Pneumonia (Trial Version 5). ${ }^{6}$

Chest CT of 11 COVID-19 patients showed multiple patchy ground-glass opacities and consolidation shadows in both lungs, most of which were distributed along the bronchial vascular bundle and subpleural space, with a thickened leaflet interval and thickened lobular septum, showing "crazy-paving pattern" and vascular bundle sign; some patients show isolated subpleural nodule shadow with halo sign, consistent with the recommendations of the Chinese Medical Association Radiology Branch (first edition) early imaging performance. ${ }^{7}$ There were no large consolidation images, widely distributed grid-shaped ground-glass opacities similar to the white lung imaging findings in the 11 confirmed patients. Most of the patients' 
current image characteristics were about 1 to 5 days after the onset of disease. A Retrospective analysis by Bernheim et $\mathrm{al}^{9}$ on chest CT in 121 patients with COVID-19 also showed the common CT findings in relationship to the time between symptom onset and the initial CT scan: $56 \%$ of patients could have normal chest CT in the early stage of onset (day 0-1). When the patient is diagnosed, he will be transferred to the designated hospital for further treatment, so the follow-up imaging data is unknown.

Although chest CT has an important value as a clinical imaging screening index, it has certain limitations in terms of early diagnosis, differential diagnosis, exclusion diagnosis, and final diagnosis, and it cannot replace the pathogenic diagnosis. ${ }^{10}$ This study showed that the clinical features and imaging features of early neocoronary pneumonia were not characteristic after imaging manifestations of general clinical features of suspected and confirmed patients in Shanghai, China. In addition to the general inflammatory response images, non-COVID-19 patients also presented along the subpleural surface, ground-glass opacities or isolated subpleural nodules with halo sign and other characteristics, which are difficult to distinguish from patients infected with COVID-19 and therefore, pathogenic detection is necessary as soon as possible.

In addition, the amount of virus may be different in the body of patients with different courses and conditions. In other words, although a patient may have the viral infection, because no virus is collected at the relevant site or the amount of virus collected is too small, it cannot be detected by existing methods. Therefore, for patients with high clinical suspicion, specimens should be continuously collected for multiple tests. ${ }^{11}$

In conclusion, in this single-center case report, 11 (12.8\%) of the 86 screened patients were confirmed as COVID-19 pneumonia. Analysis of the clinical characteristics of COVID-19 and non-COVID-19 indicated that the clinical characteristics of early COVID-19 patients were not specific. In addition, although chest CT findings are important to a certain extent, when there is GGO combined with bronchiectasis distributed under the pleura, or halo nodules distributed under the pleura with focal GGO, consolidation, bronchiectasis, vascular bundle sign and other imaging manifestations, it is difficult to distinguish COVID-19 from other patients in non-outbreak areas, such as Shanghai, China. Therefore, pathogenic testing should be performed as soon as possible.

\section{Authors' Contribution}

LP, KYL and CZ conceived and coordinated the study, designed, performed, analyzed the experiments and wrote the paper. FX, YFM carried out the data collection, data analysis, and revised the paper.
All authors reviewed the results and approved the final version of the manuscript.

\section{Conflict of Interest Disclosures}

The authors declare that they have no conflict of interest.

\section{Ethical Statement}

Informed consent was obtained from all individual participants included in the study.

\section{Funding Sources}

This work was supported by the Key Discipline of Pudong New Area, Shanghai (No: PWZxk2017-22).

\section{References}

1. World Health Organization. Director-General's remarks at the media briefing on 2019-nCoV on 11 February 2020. Available from: https://www.who.int/emergencies/diseases/novelcoronavirus-2019/events-as-they-happen.

2. Hernández JC, Austin Ramzy T. China Confirms New Coronavirus Spreads from Humans to Humans. Available from: https://www.nytimes.com/2020/01/20/world/asia/ coronavirus-chinasymptoms. Accessed 24 January 2020.

3. Wei Q, Wang $\mathrm{YH}, \mathrm{Ma} J \mathrm{C}$, Han J, Jiang MG, Zhao L, et al. Description of the first strain of 2019-nCoV, C-Tan-nCoV Wuhan Strain - National Pathogen Resource Center, China, 2020. Available from: http://weekly.chinacdc.cn/en/article/id/ e3a460f1-661b-4180-b562-ecd8e9502082.

4. Dong N, Yang X, Ye L, Chen KC, Chan WC, Yang MS, et al. Genomic and protein structure modelling analysis depicts the origin and infectivity of 2019-nCoV, a new coronavirus which caused a pneumonia outbreak in Wuhan, China[J/OL]. bioRxiv. January 22, 2020. doi: 10.1101/2020.01.20.913368.

5. Lu R, Zhao X, Li J, Niu P, Yang B, Wu H, et al. Genomic characterisation and epidemiology of 2019 novel coronavirus: implications for virus origins and receptor binding. Lancet. 2020. doi: 10.1016/S0140-6736(20)30251-8.

6. China National Health Commission. Diagnosis and treatment of pneumonia caused by new coronavirus infection (version 5) (Feb 8, 2020). Beijing: China National Health Commission; 2020.

7. Chinese Society of Radiology. Radiological diagnosis of new coronavirus infected pneumonitis: Expert recommendation from the Chinese Society of Radiology (First edition). Chin J Radiol. 2020;54(00):E001. doi: 10.3760/ cma.j.issn.1005-1201.2020.0001.

8. Chan JF, Yuan S, Kok KH, To KK, Chu H, Yang J, et al. A familial cluster of pneumonia associated with the 2019 novel coronavirus indicating person-to-person transmission: a study of a family cluster. Lancet. 2020;395(10223):514-523. doi: 10.1016/S0140-6736(20)30154-9.

9. Bernheim A, Mei X, Huang M, Yang Y, Fayad ZA, Zhang $\mathrm{N}$, et al. Chest CT Findings in Coronavirus Disease-19 (COVID-19): Relationship to Duration of Infection. Radiology. 2020:200463. doi: 10.1148/radiol.2020200463.

10. Huang L, Han R, Yu PG, Wnag SK, Xia LM, Correlation between $\mathrm{CT}$ and clinical manifestations of different clinical types of new coronavirus pneumonia. Chinese J Radiol. 2020;54. doi: 10.3760/cma.j.issn.1005-1201.2020.0003.

11. Wang CB. Nucleic acid test is used to analyze the cause of low positive rate of novel coronavirus pneumonia. Chinese Med J. 2020;100(00):E010. doi: 10.3760/ cma.j.cn112137-20200213-00280. 